\title{
Electrostatic quasi-monochromatic waves in the downstream region of the Earth's bow shock based on Geotail observations
}

\author{
Koichi Shin ${ }^{1}$, Hirotsugu Kojima ${ }^{1}$, Hiroshi Matsumoto ${ }^{1 *}$, and Toshifumi Mukai ${ }^{2}$ \\ ${ }^{1}$ Research Institute for Sustainable Humanosphere, Kyoto University, Gokasho, Uji, Kyoto 611-0011, Japan \\ ${ }^{2}$ Institute of Space and Astronautical Science, Japan Aerospace Exploration Agency, \\ 3-1-1 Yoshinodai, Sagamihara, Kanagawa 229-8510, Japan
}

(Received November 25, 2005; Revised September 7, 2006; Accepted September 21, 2006; Online published March 21, 2007)

\begin{abstract}
Geotail plasma wave observations show the existence of intense electrostatic quasi-monochromatic (EQM) waves in the downstream region of the Earth's bow shock. They oscillate parallel to the ambient magnetic field and appear at frequencies between the electron plasma and ion plasma frequencies. Although these waves have been believed to be Doppler-shifted ion acoustic waves, the typical plasma parameters observed in the downstream region do not support the generation conditions for ion acoustic waves. In this paper, the existence of cold electron beam-like components accompanying EQM waves is considered based on waveform and statistical analyses. Linear dispersion analyses using realistic plasma parameters revealed that the cold electron beams cause destabilization of electron acoustic waves at frequencies consistent with those of observed EQM waves. The results of observations and linear analyses suggest that EQM waves are generated by the destabilization of the electron acoustic mode.
\end{abstract}

Key words: Electrostatic plasma wave, electron acoustic mode, downstream region of the bow shock.

\section{Introduction}

The Earth's bow shock and its downstream region are highly turbulent. Various plasma waves can be observed over a wide frequency range in these regions, and data obtained by numerous spacecraft missions have been employed to study plasma wave generation around the bow shock (e.g., Fredricks et al., 1970; Rodriguez and Gurnett, 1975). Rodriguez and Gurnett (1975) reported three types of electrostatic plasma waves observed by the IMP 6 satellite in the near-Earth magnetosheath region-broadband electrostatic noise (BEN), narrowband electrostatic emissions, and electron plasma waves. Among these three types of electrostatic plasma waves, narrowband electrostatic emissions are most frequently observed in the downstream region of the Earth's bow shock. The frequency range of these waves is between the electron plasma frequency $\left(f_{\mathrm{pe}}\right)$ and the ion plasma frequency $\left(f_{\mathrm{pi}}\right)$. Shin et al. (2005) analyzed the waveforms of these narrowband electrostatic emissions using the plasma wave instrument (PWI) onboard the Geotail spacecraft and showed that the waveforms are almost sinusoidal with the wave normal parallel to the ambient magnetic field. These waves were termed electrostatic quasi-monochromatic (EQM) waves after their waveforms.

The results of observational and theoretical studies over recent decades suggest that the most plausible wave mode

\footnotetext{
*Now at Kyoto University, Yoshida-Honmachi, Sakyo, Kyoto 6068501, Japan.

Copyright (c) The Society of Geomagnetism and Earth, Planetary and Space Sciences (SGEPSS); The Seismological Society of Japan; The Volcanological Society of Japan; The Geodetic Society of Japan; The Japanese Society for Planetary Sciences; TERRAPUB.
}

responsible for EQM waves is the Doppler-shifted ion acoustic wave mode (e.g., Rodriguez, 1979; Gallagher, 1985). However, the temperature ratio of electrons to ions in the downstream region of the Earth's bow shock is typically less than or equal to $1\left(T_{\mathrm{e}} / T_{\mathrm{i}} \lesssim 1\right)$, which is not consistent with the theoretical condition $\left(T_{\mathrm{e}} / T_{\mathrm{i}} \gg 1\right)$ required for the generation of ion acoustic waves. This discrepancy casts doubt on the generation model of EQM waves involving the Doppler-shifted ion acoustic wave. Several previous studies have suggested that Doppler-shifted ion acoustic waves are generated in the bow shock or ion foreshock regions and that they are convected into the downstream region of the bow shock. However, since the ion acoustic mode is purely electrostatic, it is likely to be quickly damped and is not expected to propagate far from the generation region. Consequently the generation of narrowband electrostatic emissions in the downstream region of the bow shock remain to be explained comprehensively.

In the present paper, EQM waves in the downstream region of the bow shock are investigated using plasma wave and particle data obtained by the Geotail spacecraft. The electron beam-like components accompanying EQM waves were examined (Shin et al., 2005). A statistical analysis is performed to clarify the spatial distribution of EQM waves, and this yields important information concerning the generation mechanism of EQM waves. A linear analysis of EQM waves is also conducted using plasma parameters observed by the Geotail spacecraft. Based on the present investigation, it is proposed that EQM waves are generated by the destabilization of the electron acoustic mode due to the existence of drifted electron populations with different temperatures. 
(a)

GEOTAIL PWI MCA 19950115

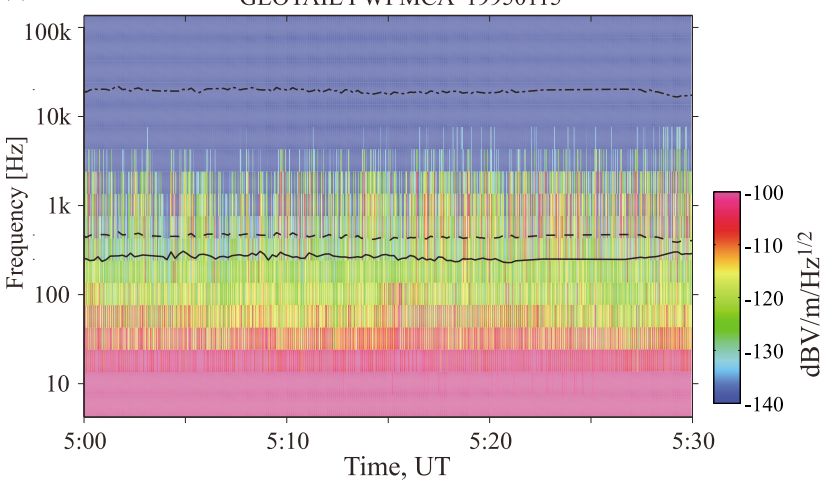

(b)

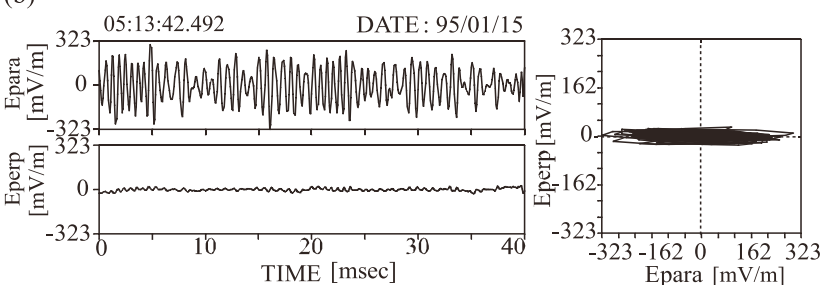

Fig. 1. (a) Dynamic spectrum of EQM waves in the downstream region of the Earth's bow shock observed on January 15, 1995 by the MCA onboard PWI. Solid, dashed and dot-dashed lines represent electron cyclotron, ion plasma, and electron plasma frequencies. (b) Waveform (left) and hodograph (right) of EQM waves. The Geotail spacecraft is located at $\left(X_{G S E}, Y_{G S E}, Z_{G S E}\right)=\left(-0.43,20.90,-1.14 R_{\mathrm{E}}\right)$, and the ambient magnetic field is $\left(B_{0 X}, B_{0 Y}, B_{0 Z}\right)=(-8.13,8.57,3.07 \mathrm{nT})$.

(a)

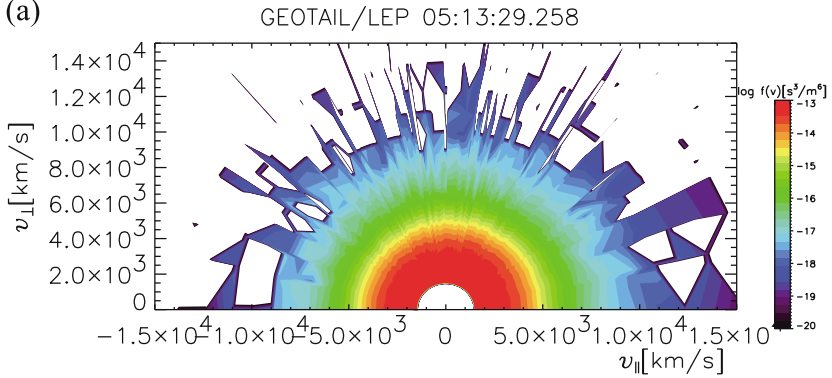

(b)

GEOTAIL/LEP 05:13:29.258

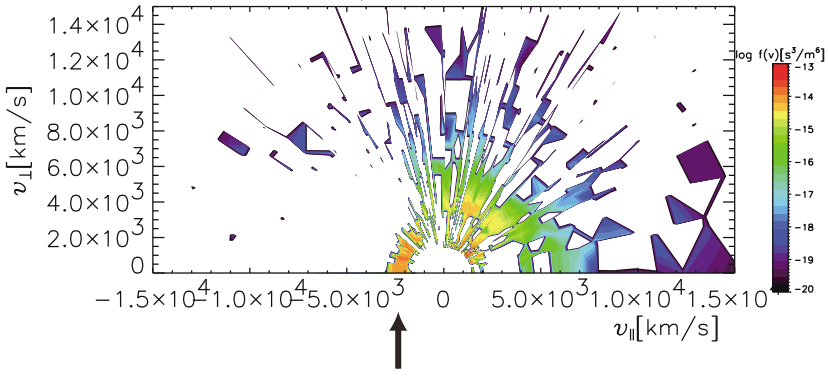

Fig. 2. (a) Electron velocity distribution for January 15, 1995 for the period from 5:13:29 UT to 5:13:41 UT. (b) Anisotropic component of electron velocity distribution. Electron beam-like component is indicated by an arrow. Phase densities are displayed as color contours, and positive velocity is defined as the ambient magnetic field direction.

\section{Observations}

\subsection{Geotail observations}

Shin et al. (2005) reported the characteristics of EQM waves observed in the downstream region of the bow shock based on Geotail waveform observations. Figure 1(a) shows the frequency-time spectrogram of the observed electric (a)
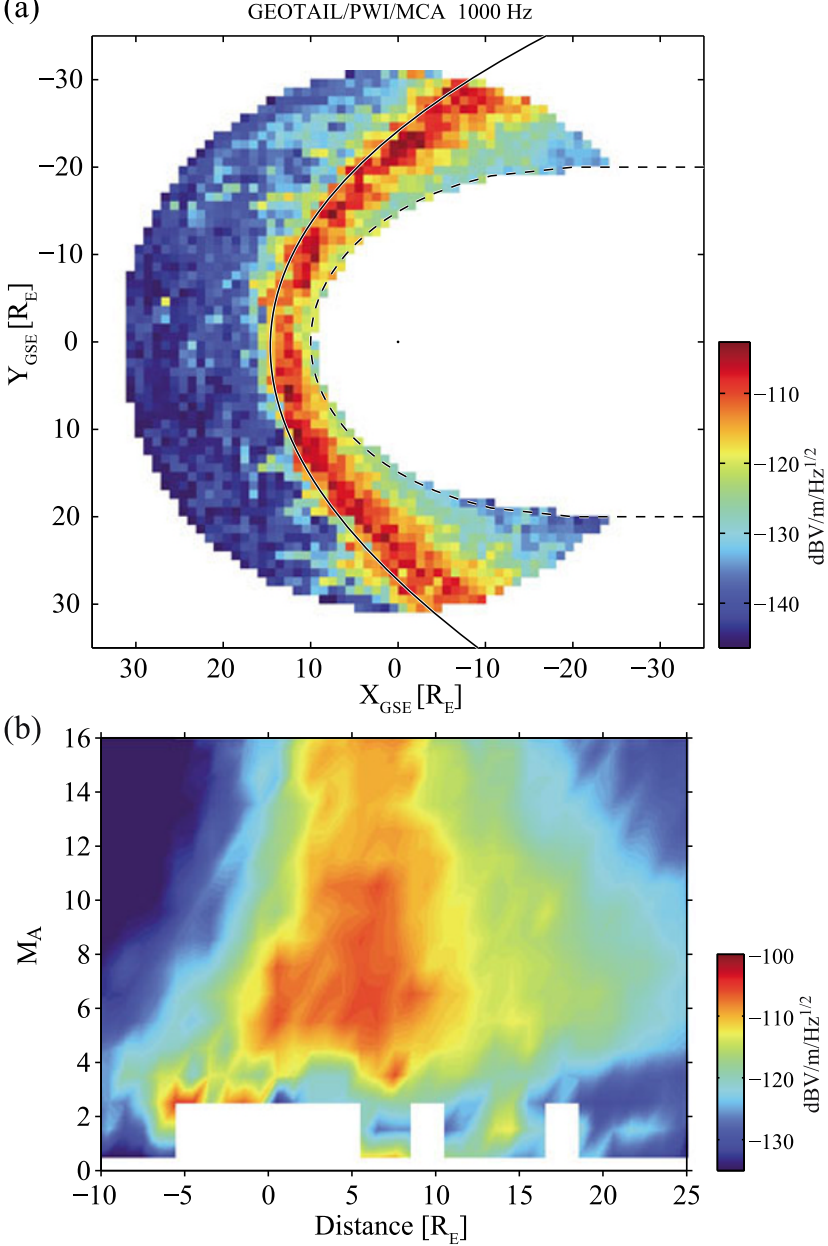

Fig. 3. (a) Spatial distribution of averaged electric field intensity on the $1-\mathrm{kHz}$ MCA channel projected onto the ecliptic plane. Spatial resolution is $1 R_{\mathrm{E}}$. Solid and dotted lines denote the averaged location of bow shock and magnetopause. (b) Dependence on the distance from the bow shock and Alfven Mach number of electric field intensities (color contour) on the 1-kHz MCA channel. The data shown are averages for the period from March 1, 1995 to December 31, 1999.

fields from 05:00 UT to 05:30 UT January 15, 1995. In this period, the Geotail spacecraft remained in the downstream region of the bow shock. The spectra were generated from data with high temporal resolution obtained by the multi channel analyzer (MCA) receiver of the Geotail PWI (Matsumoto et al., 1994). Solid, dashed and dot-dashed lines shown in Fig. 1(a) represent electron cyclotron, ion plasma and electron plasma frequencies. Figure 1(b) shows the waveforms of the electric fields observed by the waveform capture (WFC) receiver for $40 \mathrm{~ms}$ from 05:13:42.492 UT at $\left(X_{G S E}, Y_{G S E}, Z_{G S E}\right)=\left(-0.43,20.90,-1.14 R_{\mathrm{E}}\right)$, along with polarization on the $E_{\|}-E_{\perp}$ plane defined relative to the ambient magnetic field. Here, subscript GSE means the Geocentric Solar Ecliptic coordinate system, and $R_{\mathrm{E}}$ denotes the Earth radius. Since the two pairs of electric field sensors on the Geotail spacecraft are located on the spin plane, sub-coincident with the ecliptic plane, the polarization shown is the projection on the spin plane. Intense narrowband spectra also appear at frequencies close to $1 \mathrm{kHz}$, between the ion plasma frequency and the electron plasma frequency. The observed waveforms are quasi- 
monochromatic, with electric field orientation sub-parallel to the ambient magnetic field. Since the EQM waves are not accompanied by magnetic field components, the EQM waves are purely electrostatic, with wave normal oriented parallel to the ambient magnetic field.

Shin et al. (2005) reported that EQM waves are accompanied by electron beam-like components. Figure 2 shows the electron velocity distribution observed by the low energy particle (LEP) experiment (Mukai et al., 1994) of Geotail for the period from 5:13:29 UT to 5:13:41 UT January 15,1995 . To clarify the existence of an electron beam, the electron velocity distribution was divided into isotropic and anisotropic components using the method proposed by Omura et al. (1999). Figure 2 (a) shows the full electron velocity distribution function in the $v_{\|}-v_{\perp}$ plane, and (b) shows the anisotropic component of the electron velocity distribution. Phase densities are displayed as color contours, and positive velocity is defined as the ambient magnetic field direction. The existence of a beam-like component (displayed by the arrow) can be seen at velocities near $2000 \mathrm{~km} / \mathrm{s}$ in the direction anti-parallel to the ambient magnetic field, directed towards the downstream region away from the bow shock. Since the orientation of the electron beam-like components is always downstream, it is likely that the beam represents a flow of electrons accelerated in the transition region of the bow shock. The coexistence of cold electron beam-like components and hot background electrons suggest that the EQM waves are generated by the destabilization of the electron acoustic mode affected by the presence of electron populations with different temperatures.

\subsection{Spatial distribution of EQM waves}

Additional results concerning the relationship between the generation of EQM waves and the electron beam-like components accelerated in the bow shock are obtained by a statistical analysis. Since the EQM waves are observed at frequencies near $1 \mathrm{kHz}$, the intensities of the electric field spectrum data from the $1-\mathrm{kHz}$ MCA channel are used for analysis.

Figure 3(a) shows the spatial distribution of electric field intensities projected onto the ecliptic plane in the GSE coordinate system. The data shown are averages for the period from March 1, 1995 to December 31, 1999. Spatial resolution is $1 R_{\mathrm{E}}$. Solid and dotted lines denote the averaged location of bow shock and magnetopause (Fairfield, 1971). Data observed in the magnetosphere are eliminated from the data set. Intense EQM waves can be seen to be distributed along the bow shock and in its downstream region. A difference in intensity between the upstream and downstream regions of the bow shock can clearly be seen. Further, the intensities of the EQM waves tend to decrease with increasing distance from the bow shock. The waveform and statistical analyses confirm that EQM waves occur in the downstream region of the bow shock. The gradual decrease in EQM wave intensity with distance from the bow shock suggests that the free energy sources of the EQM waves are distributed around the bow shock.

Dependence of EQM wave intensities on the distance from the bow shock and Alfven Mach number $\left(M_{A}\right)$ are shown in Fig. 3(b). Here the distance means the distance from the space craft to the bow shock along the ambient magnetic field at the observation point. The intensities of the EQM waves gradually decreases with distance from the bow shock. The intensities and observation area peak around $M_{A} \sim 6$ in the Alfven Mach number dependence and tend to decrease with increasing the Alfven Mach number for $M_{A}>6$. These tendencies are very similar to the numerical simulation results obtained by Leroy et al. (1982), who examined the Alfven Mach number dependence of the electric potential overshoot by the computer simulation for the bow shock. They showed that the shock potentials are formed most efficiently in the $M_{A} \sim 6$. Since in our model electrons accelerated in the bow shock lead to the generation of electron beam-like components, which destabilize the EQM waves, the numerical simulation results by Leroy et al. (1982) are very consistent with our observated result.

\section{Electron Acoustic Mode}

EQM waves populate a characteristic frequency range near $1 \mathrm{kHz}$, which corresponds to the range from the ion plasma frequency to the electron plasma frequency, and the electric field of the EQM waves is oriented parallel to the ambient magnetic field. The most plausible wave mode of electrostatic waves with these characteristics is the electron acoustic mode, which is a normal mode for plasmas containing two electron components of different temperatures (Watanabe and Taniuti, 1977; Gary and Tokar, 1984). The electron acoustic mode can be driven when in resonance with the positive slope of an electron beam (Tokar and Gary, 1984). To examine the stability of the electron acoustic mode, we performed a linear dispersion analysis using realistic electron velocity distributions based on observations.

The typical background electron velocity distribution in the downstream region of the bow shock is characterized by a flat top shape. The electron velocity distribution is thus described as the sum of a modified Lorentzian distribution and a Maxwellian distribution drifting in the downstream direction (Feldman et al., 1982, 1983). For the event of January 15,1995 , the plasma parameters which fit to the observed electron velocity distribution are $V_{\mathrm{d}}=2200 \mathrm{~km} / \mathrm{s}$, $v_{\| \mathrm{L}}=3850 \mathrm{~km} / \mathrm{s}, v_{\| \mathrm{tb}}=650 \mathrm{~km} / \mathrm{s}, n_{\mathrm{L}}=15.5 / \mathrm{cc}$ and $n_{\mathrm{b}} / n_{\mathrm{L}}=0.015$. Here, $V_{\mathrm{d}}, v_{\| \mathrm{L}}, v_{\| \mathrm{tb}}, n_{\mathrm{L}}$ and $n_{\mathrm{b}}$ are the drift velocity, parallel thermal velocity of the modified Lorentzian distribution, parallel thermal velocity of the electron beam, number density of background electrons and number density of the electron beam, respectively. Additionally, the solar wind velocity $\left(V_{\mathrm{sw}}\right)$ and the ion temperature obtained from the LEP instrument are approximately $300 \mathrm{~km} / \mathrm{s}$ and $70 \mathrm{eV}$. Since the generation of the electron acoustic mode does not depend on the perpendicular thermal velocity of the electrons, we make use of the isotropic velocity distribution for the electrons in the present linear analysis. Thomsen et al. (1983) showed that the thermal velocity of a modified Lorentzian distribution can be treated as the thermal velocity of a Maxwellian velocity distribution under the condition $\omega / k v_{\| \mathrm{L}} \nsim 1$. Hence, the $v_{\| \mathrm{L}}$ estimated above is employed as the thermal velocity of the background Maxwellian electron velocity distribution for 

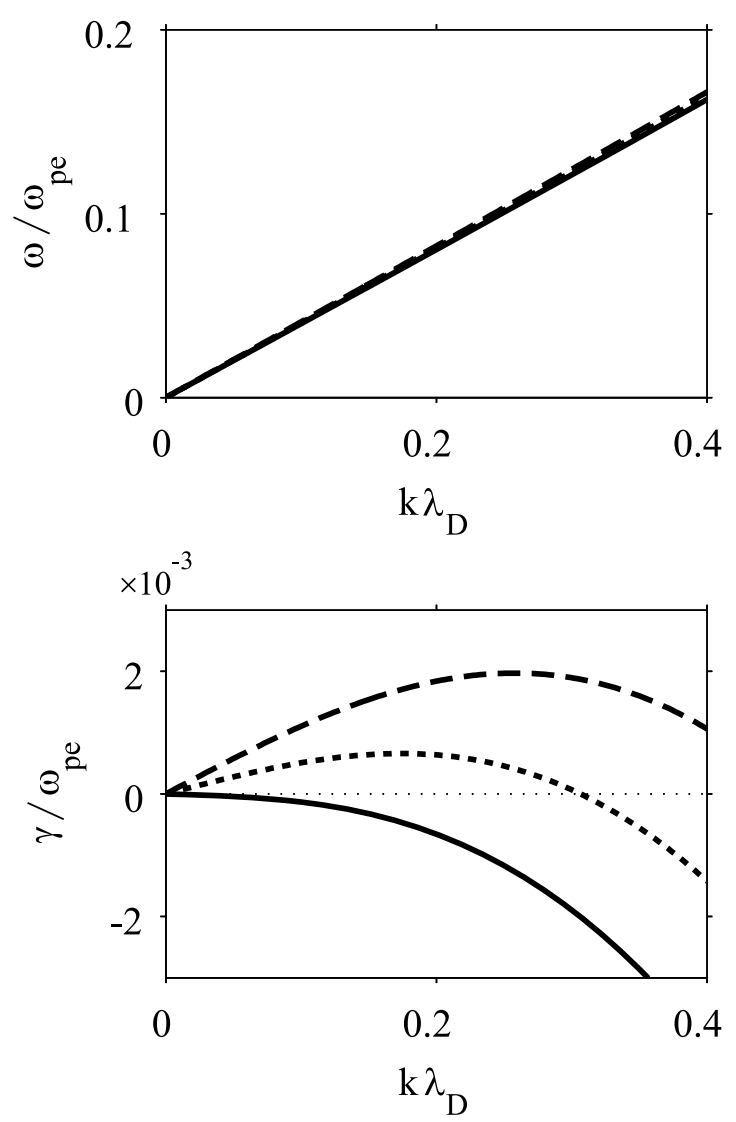

Fig. 4. Dependence of electron acoustic mode stability on the thermal velocity of the electron beam. (Upper) Wave angular frequency, (lower) growth rate normalized with respect to electron plasma angular frequency. Horizontal axis denotes the wave number normalized by a Debye length of the electron acoustic mode. Dashed, dotted and solid lines represent electron beam parallel thermal velocities of 240,260 and $280 \mathrm{~km} / \mathrm{s}$, respectively.

linear dispersion analysis. Linear dispersion analysis with these parameters was conducted using the KUPDAP program developed by Kyoto University.

Figure 4 shows the dependence of the stability of the electron acoustic mode on the thermal velocity of the electron beam. The top and bottom panel of Fig. 4 show the wave number normalized by the Debye length versus wave angular frequency and growth rate normalized by the electron plasma angular frequency. Dashed, dotted and solid lines show the results in cases of $240 \mathrm{~km} / \mathrm{s}, 260 \mathrm{~km} / \mathrm{s}$ and $280 \mathrm{~km} / \mathrm{s}$, respectively, for the thermal velocity of the electron beam. These results show that the electron acoustic mode becomes unstable as the parallel thermal velocity of the electron beam reaches $260 \mathrm{~km} / \mathrm{s}$ or lower. The dependence of electron acoustic mode stability on the density of the electron beam is shown in Fig. 5 assuming a parallel thermal velocity of $260 \mathrm{~km} / \mathrm{s}$. Dashed, dotted and solid lines denote results with electron beam densities normalized by the number density of background electrons $\left(n_{\mathrm{b}} / n_{\mathrm{L}}\right)$ of $0.012,0.015$ and 0.018 , respectively. Under these conditions, the electron acoustic mode becomes unstable when the density of $n_{\mathrm{b}} / n_{\mathrm{L}}$ reaches 0.015 or higher.

In the present linear analysis, the electron acoustic mode does not exhibit a positive growth rate in the case of $v_{\| \mathrm{tb}}=$ $650 \mathrm{~km} / \mathrm{s}$, which is the equivalent value obtained from the
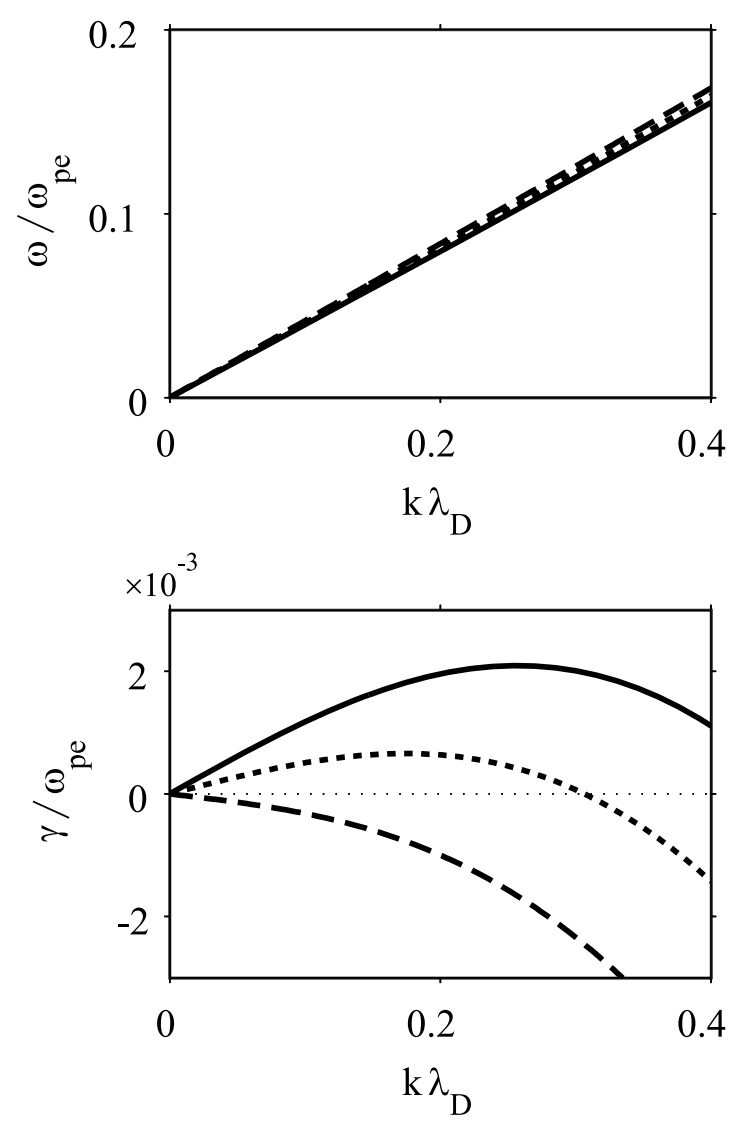

Fig. 5. Dependence of the stability of the electron acoustic mode on the number density of the electron beam normalized with respect to the thermal velocity of the background electrons, assuming an electron beam thermal velocity of $260 \mathrm{~km} / \mathrm{s}$. Dashed, dotted and solid lines denote results that represent electron beam densities normalized by the number density of background electrons of $0.012,0.015$ and 0.018 , respectively.

observed electron velocity distributions. However, since the electron acoustic mode is purely electrostatic, it is expected that excited waves would quickly thermalize the free-energy electrons in the direction parallel to the ambient magnetic field. Therefore, the assumption using initially colder electron beams is reasonable for the generation of the electron acoustic mode.

\section{Ion Acoustic Mode}

It has been suggested previously that EQM waves are Doppler-shifted ion acoustic waves (e.g., Rodriguez, 1979; Gallagher, 1985). However, the typically observed electronto-ion temperature ratio $T_{\mathrm{e}} / T_{\mathrm{i}}$ in the downstream region is less than or equal to unity, which is not consistent with the theoretical generation conditions for ion acoustic waves, which requires $T_{\mathrm{e}} / T_{\mathrm{i}} \gg 1$.

To examine this situation further, the maximum frequency of the Doppler-shifted ion acoustic wave is estimated based on the plasma parameters observed during the period shown in Fig. 1(b). The equation for the frequency of the Doppler-shifted ion acoustic wave is expressed as

$$
f_{\text {Dia }}=\left|f_{\text {ia }}\left(1+\frac{v_{\text {sw }} \cos \theta}{v_{\text {ia }}}\right)\right|
$$

where the ion plasma frequency $\left(f_{\mathrm{pi}}\right)$ is $930 \mathrm{~Hz}$, the ion 


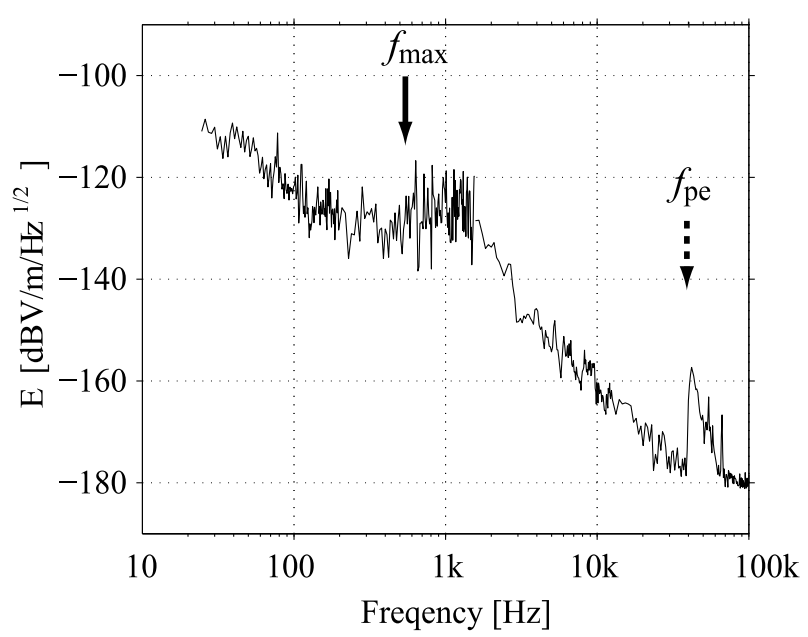

Fig. 6. Electric field spectrum (5 min average) for EQM waves observed during the period from 5:10 UT to 5:15 UT, January 15, 1995 (SFA data). Solid arrow denotes maximum frequency of the Doppler-shifted ion acoustic wave $\left(f_{\max }\right)$, and dotted arrows denote the electron plasma frequency.

acoustic velocity $\left(v_{\text {ia }}\right)$ is $167 \mathrm{~km} / \mathrm{s}$, the solar wind velocity $\left(v_{\mathrm{sw}}\right)$ is $300 \mathrm{~km} / \mathrm{s}$ and the angle between the solar wind flow vector and the propagation vector of the ion acoustic wave $(\theta)$ is $150^{\circ}$. Since frequencies of ion acoustic waves $\left(f_{\text {ia }}\right)$ are below the ion plasma frequency, we can calculate the maximum Doppler-shifted ion acoustic wave frequency using $f_{\mathrm{ia}}=f_{\mathrm{pi}}$.

The maximum Doppler-shifted ion acoustic wave frequency thus estimated is $543 \mathrm{~Hz}$. If the EQM waves are ion acoustic waves, the frequency of observed EQM waves therefore should not exceed $543 \mathrm{~Hz}$. Figure 6 shows the electric field spectrum of the EQM waves (5-min average) for the period from 5:10 UT to 5:15 UT January 15, 1995 and the maximum frequency of the Doppler-shifted ion acoustic waves (displayed by the solid arrow). The electric field enhancement near $1 \mathrm{kHz}$ corresponds to the EQM wave. The maximum frequency of the Doppler-shifted ion acoustic wave is clearly lower than the frequency of the EQM wave. In order to increase the frequency of the ion acoustic wave to match that observed for the EQM wave, the solar wind velocity must be substantially higher. It is thus concluded that the EQM waves observed in this event are not Doppler-shifted ion acoustic waves.

When the solar wind vector projected onto the ambient magnetic field is oriented parallel to the propagation vector of the ion acoustic wave, the estimated frequency of the Doppler-shifted ion acoustic wave is consistent with the frequency of the EQM wave. On the contrary, the Doppler shift effect lowers the frequency of the ion acoustic wave when the solar wind vector projected onto the ambient magnetic field is oriented anti-parallel to the propagation vector of the ion acoustic wave, as is the case in the present observed event. The estimated frequency of the Dopplershifted ion acoustic wave is thus not consistent with the frequency of the EQM wave for this event.

Seven events in which EQM waves are accompanied by electron beam-like components are analyzed in this manner. If the EQM wave is a Doppler-shifted ion acoustic wave,
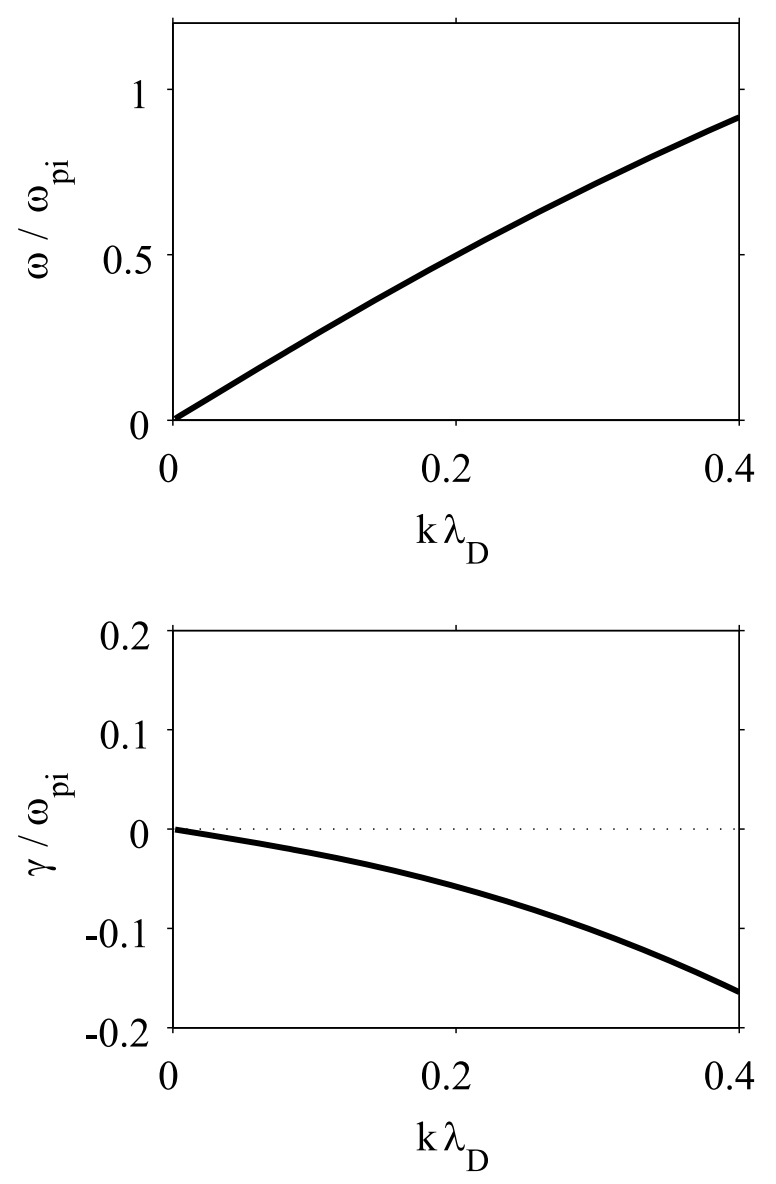

Fig. 7. Wave angular frequency (upper) and growth rate (lower) normalized with respect to the ion plasma angular frequency as a function of wave number of the ion acoustic mode assuming thermal velocity of $700 \mathrm{~km} / \mathrm{s}$ and density of $n_{\mathrm{b}} / n_{\mathrm{L}}=0.15$.

the argument above suggests that the frequency of the EQM wave will be dependent on the solar wind velocity and the angle between the solar wind vector and the propagation vector of the EQM wave. However, in all of the cases examined, the frequencies of the EQM waves are higher than the ion plasma frequencies, regardless of the angle between the solar wind vector and the propagation vector of the EQM wave. It is thus fair to conclude that the EQM wave is not a Doppler-shifted ion acoustic wave.

\section{Discussion and Conclusions}

The generation of EQM waves in the downstream region of the Earth's bow shock was examined on the basis of Geotail plasma wave and particle observations coupled with statistical and linear dispersion analyses. Statistical analysis confirmed that EQM waves are closely related to the shock potentials which accelerate electrons, resulting in the formation of electron beam-like components, while linear dispersion analysis using realistic plasma parameters revealed that the electron beams with the different temperature from those of background electrons destabilize the electron acoustic mode.

Although the most plausible wave mode responsible for EQM waves has been believed to be the Doppler-shifted ion acoustic wave mode, the typical plasma parameters observed in the downstream region do not support the gen- 
eration conditions for ion acoustic waves. Further, the expected frequency of ion acoustic mode is substantially lower than that of the observed EQM wave. Additionally, we perform the linear dispersion analysis for the ion acoustic mode using the same plasma parameters as those for the electron acoustic mode. The top and bottom panel of Fig. 7 show the wave angular frequency and growth rate normalized with respect to the ion plasma angular frequency as a function of wave number of the ion acoustic mode assuming thermal velocity of $700 \mathrm{~km} / \mathrm{s}$ and density of $n_{\mathrm{b}} / n_{\mathrm{L}}=0.15$. Although the density of the electron beam for the ion acoustic mode is approximately tenfold higher than that for the electron acoustic mode, the ion acoustic mode exhibits no growth. The destabilization of the ion acoustic mode is therefore not expected in the downstream region of the bow shock.

The electron acoustic wave was discussed as an alternative generation mechanism for EQM waves. Assuming an electron beam that is two- to threefold colder than that obtained by the observed electron velocity distribution, the electron acoustic wave can be destabilized at the same frequencies as the EQM waves. The observed electron velocity distribution may thus occur as a result of the generation of EQM waves, which causes thermalization of the electron beams. Similar types of electron velocity distributions have been observed by the VELA 4 (Montgomery et al., 1970) and ISEE 2 spacecraft (Feldman et al., 1982, 1983) in the transition region of the bow shock. The direction and drift velocity of electron beam-like components shown in Fig. 2 agree with the ISEE 2 observations. Since the electron beam-like components are oriented downstream away from the bow shock, it is possible that the electron beamlike components observed in the downstream region of the bow shock are accelerated in the bow shock by the crossfield electrostatic potential. Based on realistic parameters observed in the bow shock transition by the ISEE 2 spacecraft, Thomsen et al. (1983) suggested the possibility that both ion acoustic waves and electron acoustic waves are destabilized by the two electron populations (beam and flattopped distribution) and an ion component. Meanwhile, in the downstream region of the bow shock, we showed EQM waves are generated by the destabilization of the electron acoustic mode.

Although we have a limited data set due to the normal observation mode of science instruments onboard the Geotail spacecraft, we have been able to adequately discuss in this paper the generation of EQM waves in the downstream region of the Earth's bow shock, and results of statistical and linear dispersion analysis show that the most plausible wave mode of the EQM wave can be considered to be the electron acoustic wave mode due to the existence of electron beams accelerated in the bow shock.
Acknowledgments. The authors would like to thank the ISAS/NASA Geotail mission project team for valuable support. We thank T. Sugiyama for helpful discussions on the analyses of plasma data in the downstream region. This work was supported by JSPS KAKENHI (15204044).

\section{References}

Fairfield, D. H., Average and unusual locations of the earth's magnetopause and bowshock, J. Geophys. Res., 76, 6700-6716, 1971.

Feldman, W. C., S. J. Bame, S. P. Gary, J. T. Gosling, D. J. McComas, M. F. Thomsen, G. Paschmann, N. Sckopke, M. M. Hoppe, and C. T. Russell, Electron heating within the earth's bow shock, Phys. Rev. Lett., 49, 199, 1982.

Feldman, W. C., R. C. Anderson, S. J. Bame, S. P. Gary, J. T. Gosling, D. J. McComas, M. F. Thomsen, G. Paschmann, and M. M. Hoppe, Electron velocity distribution near the earth's bow shock, J. Geophys. Res., 88 , 96, 1983.

Fredricks, S. A., G. M. Crook, C. F. Kennel, I. M. Green, F. L. Scarf, P. J. Coleman, and C. T. Russell, OGO 5 observations of electrostatic turbulence in bow shock magnetic structures, J. Geophys. Res., 75, $3751,1970$.

Gallagher, D. L., Short-wavelength electrostatic waves in the earth's magnetosheath, J. Geophys. Res., 90, 1435-1448, 1985.

Gary, S. P. and R. L. Tokar, The electron acoustic mode in a two temperature plasma, Phys. Fluids, 28, 2439, 1984.

Leroy, M. M., D. Winske, C. C. Goodrich, C. S. Wu, and K. Papadopoulos, The structure of perpendicular bow shocks, J. Geophys. Res., 87, 50815094, 1982

Matsumoto, H., I. Nagano, R. R. Anderson, H. Kojima, K. Hashimoto, M. Tsutsui, T. Okada, I. Kimura, Y. Omura, and M. Okada, Plasma wave observations with GEOTAIL spacecraft, J. Geomag. Geoelectr., 46, 59-95, 1994.

Montgomery, M. D., J. R. Asbridge, and S. J. Bame, VELA 4 plasma observations near the earth's bowshock, J. Geophys. Res., 75, 1217, 1970

Mukai, T., S. Machida, Y. Saito, M. H. T. Terasawa, N. Kaya, T. Obara, and M. E. A. Nishida, The low energy particle (LEP) experimentonboard the GEOTAIL satellite, J. Geomag. Geoelectr., 46, 669-692, 1994.

Omura, Y., H. Kojima, N. Miki, T. Mukai, H. Matsumoto, and R. R. Anderson, Electrostatic solitary waves carried by diffused electron beams observed by the GEOTAIL spacecraft, J. Geophys. Res., 104, 14,62714,637, 1999.

Rodriguez, P., Magnetosheath electrostatic turbulence, J. Geophys. Res., 84, 917-930, 1979.

Rodriguez, P. and D. A. Gurnett, Electrostatic and electromagnetic turbulence associated with the earth's bow shock, J. Geophys. Res., 80 , 19-31, 1975.

Shin, K., H. Kojima, H. Matsumoto, and T. Mukai, Electrostatic quasimonochromatic waves downstream of the bow shock: Geotail observations, in Frontiers in Magnetospheric Physics, COSPAR Colloquia Series Vol. 16, edited by M. Hoshino, Y. Omura, and L. J. Lanzerotti, p. 293, Elsevier, Kidlington, Oxford, 2005.

Thomsen, M. F., H. C. Barr, S. P. Gary, W. C. Feldman, and T. E. Cole, Stability of electron distribution within the earth's bow shock, J. Geophys. Res., 88, 3035, 1983.

Tokar, R. L., and S. Gary, Electrostatic hiss and the beam driven electron acoustic instability in the dayside polar cusp, Geophys. Res. Lett., 11, $1180,1984$.

Watanabe, K. and T. Taniuti, Electron acoustic mode in a plasma of two temperature electrons, J. Phys. Soc. Japan, 43, 1819, 1977.

K. Shin (e-mail: shin@rish.kyoto-u.ac.jp), H. Kojima, H. Matsumoto, and T. Mukai 